\title{
Subclinical Hypothyroidism
} and Cardiovascular Mortality in Patients Suffered from Heart Failure with Preserved Ejection

\section{Fraction}

Keywords: Subclinical hypothyroidism; Heart failure with preserved ejection fraction; Cardiovascular mortality; Thyroid stimulating hormone

\begin{abstract}
Background: Subclinical hypothyroidism ( $\mathrm{SCH}$ ) is underdiagnosed in patients (pts) with heart disease, however, there has been controversy surrounding whether it is a cardiovascular risk factor or not. To determine the prognostic significance of $\mathrm{SCH}$ in Heart Failure (HF) with Preserved Ejection Fraction (HFpEF) we followed a cohort of pts for 9 years.
\end{abstract}

Methods: In this retrospective cohort, we queried our HF data base to identify HFpEF pts diagnosed in 2006 (registry initiation date) based on symptoms, BNP, and Echocardiogram with no history of hypothyroidism. Primary outcome was cardiovascular disease (CVD) mortality. Subjects divided into 2 groups based on presence or absence of SCH. SCH was defined as TSH of 5-10 mIU/L and norma free $\mathrm{T} 4$ in the absence of symptoms or hypothyroidism history.

Results: We identified 249 eligible pts, of who 71 had SCH (28.5\%) Mean age was $72.1 \pm 13.4$ years. Women constituted $56.6 \%$. Follow up was for $8.75 \pm 0.17$ years. The CVD mortality rate for SCH pts was $29.6 \%$ versus $14.9 \%$ for the others ( $\mathrm{P}=0.008)$. The median survival time for $\mathrm{SCH}$ pts was significantly lower than pts with normal thyroid function, $99.9 \pm$ 2.9 and $107.7 \pm 1.5$ months, respectively $(P=0.008$ after adjustment for age, sex, hypertension, and other risk factors) (Figure 1).

Conclusion: $\mathrm{SCH}$ is a significant predictor of mortality in HFpEF pts, despite controlling for some major risk factors. Therefore a mild asymptomatic elevation in TSH has a significant impact on outcome.

\section{Background}

Subclinical hypothyroidism (SCH) can be defined as an elevated level of thyroid-stimulating hormone (TSH) with a normal thyroxine (T4) level. The cardiovascular system is one specific target of thyroid hormones. Thyroid hormones, specifically triiodothyronine (T3), are known to have beneficial cardiovascular effects including facilitating myocardial relaxation and lowering peripheral vascular resistance. Derangement of the thyroid axis can thus lead to a spectrum of cardiovascular manifestations. SCH is underdiagnosed in patients with heart disease, however, there has been controversy surrounding whether it is a cardiovascular risk factor or not. This relationship has been studied in heart failure (HF) with reduced ejection fraction (EF), but the frequency of thyroid hormone abnormalities in heart failure with preserved EF (HFpEF) is unknown [1].
Currently, there exists a paucity of evidence on the beneficial effects of thyroxine hormone replacement on cardiovascular mortality outcomes in subclinical hypothyroidism. Also, the clinical relevance of measuring and treating supra-normal TSH levels in newly diagnosed heart failure patients with preserved ejection fraction requires further study. It has been proven beyond doubt that thyroid hormone metabolism can be altered in heart disease. Conversely, thyroid hormone dysfunction can result in altered ventricular contractility/relaxation dynamics and compromised cardiac function. This fact has important clinical implications in that thyroid dysfunction represents one of the few potentially reversible causes of heart failure [2]. Accordingly, we aimed to determine the prognostic significance of $\mathrm{SCH}$ in $\mathrm{HFpEF}$ through following a cohort of HFpEF patients for 9 years.

\section{Patients and Methods}

This study was conducted following the approval of advocate health care Institutional Review Board. In this retrospective cohort, our institutional heart failure database was reviewed. All patients HFpEF diagnosis on 2006 were included. All patients with confirmed HFpEF diagnosis based on symptoms, brain natriuretic peptide (BNP), and transthoracic echocardiogram were included. HFpEF patients were identified as patients with symptoms/signs of heart failure with elevated BNP and an ejection fraction of $50 \%$ or more.

Primary outcome was cardiovascular disease (CVD) mortality. Subjects divided into 2 groups based on presence or absence of SCH. $\mathrm{SCH}$ was defined as TSH of 5-10 mIU/L and normal free T4 in the absence of symptoms or hypothyroidism history.

\section{Statistical Analysis}

All data including patient's demographics, medical history, medications, clinical presentation, ECG, and echocardiographic details were obtained from advocate Illinois masonic clinical records and our prospectively collected databases. In addition, the source for follow-up information was subsequent clinic visits, hospital 


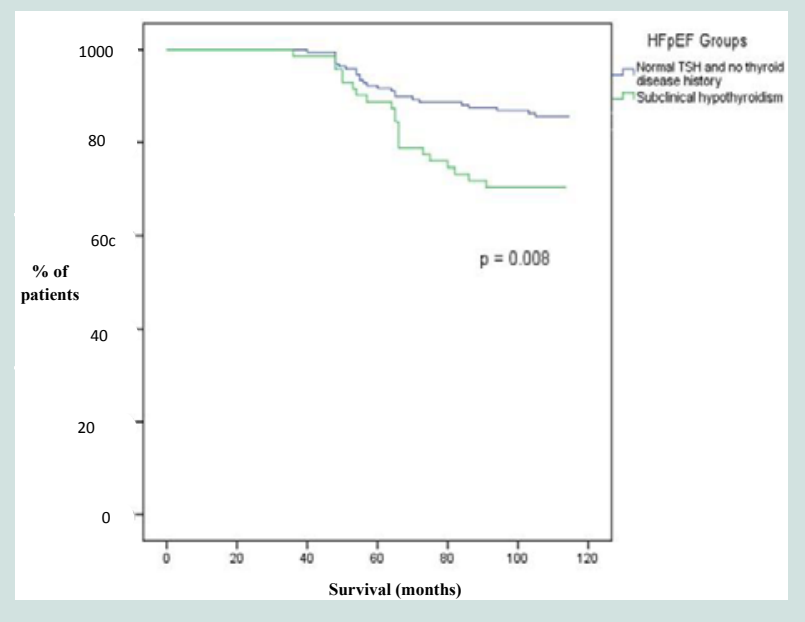

Figure 1: Kaplan Meier curve (survival curve) comparing SCH patients and the control HFpEF patients.

admissions, emergency room visits, and written correspondence from local physicians. Descriptive statistics for data with normal distribution were presented as means and standard deviations from the mean, while any other type of data distribution was presented using median with range.

Multivariate analysis and Cox regression modelling were used to assess the predictors of poor outcome. Probability value ( $p$-value) of $\leq 0.05$ was considered statistically significant.

\section{Results}

Based on the above criteria we identified 249 eligible patients, of who 71 had SCH (28.5\%). The rest of the patients where those with HFpEF diagnosis as above and normal TSH with no thyroid disease history. Mean age was $72.1 \pm 13.4$ years. Women constituted $56.6 \%$ (141 patients), while men represented $43.4 \%$ (108 patients). Mean duration of follow up was for $8.75 \pm 0.17$ years (Table 1 ). The CVD mortality rate for $\mathrm{SCH}$ patients was $29.6 \%$ versus $14.9 \%$ for the others $(\mathrm{P}=0.008)$, which indicates significant difference and higher mortality risk for patients with $\mathrm{SCH}$. The median survival time for $\mathrm{SCH}$ patients was significantly lower than patients with normal thyroid function, $99.9 \pm 2.9$ and $107.7 \pm 1.5$ months, respectively. After adjusting for some major risk factors including age, sex, hypertension, diabetes, coronary artery disease, $\mathrm{BMI}$, and atrial fibrillation, $\mathrm{SCH}$ remained a significant predictor of mortality in $\operatorname{HFpEF}(\mathrm{P}=0.008)$ (based on multivariate regression analysis) (Figure 1).

\section{Discussion}

The associations of SCH with all-cause or CVD mortality were controversial in previous studies. Several studies reported no association of SCH with death from CVD or all-cause mortality. All the reported studies focused on the presence of coronary artery disease and systolic heart failure, but none were conducted on patients with HFpEF and TSH $<10 \mathrm{mIU} / \mathrm{L}$. The pathophysiology of $\mathrm{SCH}$-induced diastolic dysfunction is not completely understood, but mostly related to loss of T3-induced genomic and non-genomic effects on the cardiac myocyte (through transcription factors and membrane iron-channels) leading to higher resistance, stiffness, and impaired diastolic filling.

The heart is particularly vulnerable to the reduction in local triiodothyronine (T3) levels given the essential rule that T3 plays in maintaining cardiac morphology and function. Moreover, thyroid hormone affects cardiac performance through both genomic and nongenomic effects. The genomic nuclear effects play a very significant role in the physiological impact of the thyroid hormone. The main mechanism is through the binding of T3 to specific nuclear thyroid hormone receptors (TRs), which are encoded by the $\alpha$ and $\beta$ c-erbA proto-oncogene families. TRs act by binding as homodimers or heterodimers to thyroid hormone response elements in the promoter region of some genes [2-5].

Rodondi et al. conducted a literature search regarding the association between subclinical hypothyroidism and coronary heart disease and each of the researchers were invited to join the thyroid study collaboration. After collecting all the information from all the 55, 287 individuals from different cohort studies they concluded that subclinical hypothyroidism is associated with an increased risk of coronary heart disease in those with higher TSH levels. Also, the risk of both CHD mortality and events, increases with higher concentrations of TSH and is significantly elevated in adults with TSH levels of $10 \mathrm{mIU} / \mathrm{L}$ or greater. On the other hand, minimal TSH elevations are not associated with an increased risk of CHD events or mortality [6]. This differs from our study in that TSH cutoff in our cohort was 5-10 mIU/L and nevertheless SCH continued to be a significant mortality predictor.

$\mathrm{Li}$ et al. found that subclinical hypo- and hyperthyroidism patients had increased all-cause mortality. However, after adjustment for the baseline variables, only subclinical hyperthyroidism, not subclinical hypothyroidism, was a predictor of all-cause mortality in dilated cardiomyopathy patients. In addition, traditional risk factors for adverse outcomes in HF and dilated cardiomyopathy, such as left ventricular (LV) diameter and LV ejection fraction, although entering the univariate Cox analysis model as the risk factors of all-cause mortality, were not independent prognosis predictors. The difference in the studied populations regarding race and sex ratio as well as the difference in the selected variables included in the Cox multivariate analysis might account for the different outcomes in these studies [7].

In a recent study, the combination of all available large prospective cohorts with 25,378 participants indicates that HF riskis higher with any abnormal TSH level regardless if lower or higher, particularly if TSH levels $\geq 10.0 \mathrm{mIU} / \mathrm{L}$ or TSH $<0.10 \mathrm{mIU} / \mathrm{L}$. For those with borderline TSH disturbances (TSH levels between 4.50-6.99 mIU/L and TSH levels between $0.10-0.44 \mathrm{mIU} / \mathrm{L}$ ), no significant difference was in HF risk [8].

Classification systems have been proposed grading subclinical hypothyroidism based on severity assessment into grade 1 (TSH levels $<10 \mathrm{mIU} / \mathrm{L}$ ) and grade $2(\mathrm{TSH}>10 \mathrm{mIU} / \mathrm{L})$ [9]. The natural history may differ significantly between the two groups with grade 2 category patients having higher cardiovascular events and at higher rates of transition to overt hypothyroidism [10]. A large prospective study involving elderly population ( $>85$ years) noted an association between grade 1 subclinical hyperthyroidism and decreased all-cause mortality [12]. This was later supported by a large retrospective cohort study which also noted lower mortality rates in grade 1 
Citation: Hassan A, Altamiranoufion A, Mahmoud S, Villines D, Mendyuk H, et al. Association between Subclinical Hypothyroidism and Cardiovascular Mortality in Patients Suffered from Heart Failure with Preserved Ejection Fraction. J Cardiobiol. 2016;4(1): 4.

ISSN: $2332-3671$

Table 1: Baseline characteristics of patients with HFpEF.

\begin{tabular}{|c|c|c|c|c|c|}
\hline \multicolumn{6}{|c|}{ Descriptive Statistics } \\
\hline & $\mathbf{N}$ & Minimum & Maximum & Mean & Std. Deviation \\
\hline Age (years) & 249 & 27 & 98 & 72.15 & 13.400 \\
\hline BMI $\left(\mathbf{k g} / \mathrm{m}^{2}\right)$ & 249 & 14.5 & 93.4 & 32.168 & 10.8590 \\
\hline Height (cm) & 249 & 2.0 & 191.0 & 164.358 & 15.1083 \\
\hline Weight (kg) & 249 & 42.3 & 852.0 & 91.335 & 57.4622 \\
\hline $\operatorname{BSA}\left(m^{2}\right)$ & 249 & 1.40 & 197.00 & 3.9776 & 17.24746 \\
\hline $\mathrm{Hb}(g / d \mathrm{l})$ & 249 & 1.5 & 16.4 & 10.972 & 2.0200 \\
\hline Het (\%) & 249 & 20.0 & 54.5 & 33.730 & 5.9931 \\
\hline Platelets ( $10^{3} / \mathrm{microliter)}$ & 249 & 31.0 & 600.0 & 226.789 & 79.3638 \\
\hline WBC (10 $10^{\prime} /$ iter $)$ & 249 & 3.3 & 20.1 & 8.730 & 3.0440 \\
\hline BNP (ng/L) & 223 & 1.1 & 5000.0 & 587.270 & 726.9906 \\
\hline $\mathrm{Cr}(\mathrm{mg} / \mathrm{dl})$ & 249 & 0.00 & 24.90 & 1.8155 & 1.82047 \\
\hline LVESd (mm) & 237 & 1 & 26 & 3.16 & 1.690 \\
\hline LVEDd (mm) & 237 & 2 & 8 & 4.56 & 0.830 \\
\hline LVWd (cm) & 226 & 0 & 3 & 1.17 & 0.300 \\
\hline IVSd(cm) & 224 & 1 & 4 & 1.25 & 0.360 \\
\hline PApressure (mmHg) & 189 & 0 & 89 & 36.16 & 17.759 \\
\hline LAsize (cm) & 236 & 1.0 & 5.9 & 3.877 & 0.8402 \\
\hline Aortic rootdiameter $(\mathrm{cm})$ & 226 & 0.0 & 4.5 & 3.193 & 0.5189 \\
\hline
\end{tabular}

LVESd: Left Ventricular End Systolic Diameter; LVEDd: Left Ventricular End Diastolic Diameter; LVWd: Left Ventricular Free Wall Thickness; IVSd: Inter Ventricular Septal Thickness.

subclinical hypothyroidism patients, suggesting a protective benefit of mildly supra normal TSH levels [11,12]. The same study observed and increase in myocardial infarction events in patients with grade 2 subclinical hypothyroidism. Similarly, an international prospective cohort study of 55287 patients reported increased CHD events and CHD related mortality in these subset of patients [11]. Individual participant data analysis did not identify statistically differing CHD outcomes across the age groups [11]. A new study by Ball $\mathrm{M}$ et al. suggested that even coronary collateral circulation can be poor in patients with high TSH and low T3 [13]. Some recent studies suggested the potential benefit of screening patients with diastolic dysfunction especially in the young age group [14].

Given all the conflicting data, it's in our opinion patients with mildly or significantly elevated TSHand signs/symptoms of heart failure should be aggressively treated despite the existing scarce evidence.

\section{Conclusion}

Mild SCH is a significant predictor of mortality in HFpEF patients, despite controlling for all major risk factors. Therefore a mild asymptomatic elevation in TSH has a significant impact on outcome, perhaps as a consequence on left ventricular stiffness.

\section{Limitations}

The study was based on only one enters experience. Socioeconomic status was not included and the correlation between metabolic factors was not assessed.

\section{References}

1. Selvaraj S, Klein I, Danzi S, Akhter N, Bonow RO, et al. (2012) Association of serum triiodothyronine with B-type natriuretic peptide and severe left ventricular diastolic dysfunction in heart failure with preserved ejection fraction. Am J Cardiol 110: 234-239.

2. Biondi B (2012) Mechanisms in endocrinology: heart failure and thyroid dysfunction. Eur J Endocrinol 167: 609-618.

3. Klein I, Danzi S (2007) Thyroid disease and the heart. Circulation 116: 17251735.

4. Fazio S, Palmieri EA, Lombardi G, Biondi B (2004) Effects of thyroid hormone on the cardiovascular system. Recent Prog Horm Res 59: 31-50.

5. Maia AL, Kim BW, Huang SA, Harney JW, Larsen PR (2005) Type 2 iodothyronine deiodinase is the major source of plasma T3 in euthyroid humans. J Clin Invest 115: 2524-2533.

6. Rodondi N, den Elzen WP, Bauer DC, Cappola AR, Razvi S, et al. (2010) Subclinical hypothyroidism and the risk of coronary heart disease and mortality. JAMA 304: 1365-1374.

7. Xiaoping L, Yang X, Wang Y, Ding L, Wang J, et al. (2014) GW25-e3416 The prevalence and prognostic effects of subclinical thyroid dysfunction in dilated cardiomyopathy patients: a single-center cohort study. JACC 64

8. Gencer B, Collet TH, Virgini V, Bauer DC, Gussekloo J, et al. (2012) Subclinical thyroid dysfunction and the risk of heart failure events: an individual participant data analysis from 6 prospective cohorts. Circulation 126: 1040-1049.

9. Surks MI, Ortiz E, Daniels GH, Sawin CT, Col NF, et al. (2004) Subclinical thyroid disease: scientific review and guidelines for diagnosis and management. JAMA 291: 228-238.

10. Rodondi N, den Elzen WP, Bauer DC, Cappola AR, Razvi S, et al. (2010) Subclinical hypothyroidism and the risk of coronary heart disease and mortality. JAMA 304: 1365-1374. 
Citation: Hassan A, Altamiranoufion A, Mahmoud S, Villines D, Mendyuk H, et al. Association between Subclinical Hypothyroidism and Cardiovascular Mortality in Patients Suffered from Heart Failure with Preserved Ejection Fraction. J Cardiobiol. 2016;4(1): 4.

ISSN: $2332-3671$

11. Huber G, Staub JJ, Meier C, Mitrache C, Guglielmetti M, et al. (2002) Prospective study of the spontaneous course of subclinical hypothyroidism: prognostic value of thyrotropin, thyroid reserve, and thyroid antibodies. J Clin Endocrinol Metab 87: 3221-3226.

12. Gussekloo J, van Exel E, de Craen AJ, Meinders AE, Frölich M, et al. (2004) Thyroid status, disability and cognitive function, and survival in old age. JAMA 292: 2591-2599.
13. Balli M, Cetin M, Taşolar H, Uysal OK, Yilmaz M, et al. (2016) The relationship between serum thyroid hormone levels, subclinical hypothyroidism, and coronary collateral circulation in patients with stable coronary artery disease. Turk Kardiyol Dern Ars 44: 130-136.

14. Zuin M, Scaranello F, Rigatelli G, Picariello C, Roncon L (2016) Left ventricular diastolic dysfunction in young patients with subclinical hypothyroidism: To screen or not to screen? To treat or not to treat? Int J Cardiol 214: 299-300. 